\title{
Bad thymic tumors often exhibit wanderlust
}

\author{
Cameron D. Wright, MD
}

From the Division of Thoracic Surgery, Massachusetts General Hospital, Boston, Mass.

Disclosures: Author has nothing to disclose with regard to commercial support.

Received for publication April 16, 2018; accepted for publication April 16, 2018; available ahead of print June 5, 2018.

Address for reprints: Cameron D. Wright, MD, Division of Thoracic Surgery, Massachusetts General Hospital, 55

Fruit St, Boston, MA 02114 (E-mail: cdwright@mgh.harvard.edu).

J Thorac Cardiovasc Surg 2018;156:834-5

$0022-5223 / \$ 36.00$

Copyright $(C) 2018$ by The American Association for Thoracic Surgery

https://doi.org/10.1016/j.jtcvs.2018.04.077

Fang and colleagues ${ }^{1}$ report a prospective multicenter observational study over 2 years on 275 patients with thymic tumors. Lymph node sampling of at least 1 lymph node station was mandated, but surgeons were encouraged to remove as many nodes as possible. Lymph node metastases were found in 15 of 275 patients $(5.5 \%)$. As expected, thymic tumors that had an advanced T category $(20 \%$ incidence of nodal metastasis) and World Health Organization (WHO) histology (18\% incidence of nodal metastasis) were associated with more lymph node metastases. A remarkable aspect of this study is the accrual of 275 patients with thymic tumors in a prospective study over just 2 years. Our Chinese colleagues have a large population to serve and regional centers of excellence in thoracic surgery, which allow them to report studies with numbers of patients never matched in Western reports. They are leading the way forward in uncommon diseases as their studies accrue large numbers of patients that then allow more accurate conclusions on treatments. With careful study design, accurate data collection, and careful analysis, Chinese surgeons will be leading the way with "big data."

As noted by Fang and colleagues, ${ }^{1}$ there are several limitations to this study. These include the limited number of thymic cancers and neuroendocrine tumors, the lack of mandating routine sampling of all relevant nodal stations, the varied surgical approaches, and the fact that neither of these 2 important variables ( $\mathrm{T}$ category and WHO histology) are usually known before resection in the typical patient.

How are we to use this study in the care of our patients? The low incidence of lymph node metastases in typical patients with thymoma and the high disease-free survival with standard surgery suggest lymph node dissection is unnecessary in smaller tumors that appear to be T1 or T2. The standard practice pattern is to resect anterior mediastinal tumors that clinically appear to be a readily resectable thymoma without a preoperative biopsy. Anterior mediastinal tumors that are clinically invasive are often biopsied first and offered induction therapy. Needle biopsies are usually accurate for thymic cancers and neuroendocrine tumors,

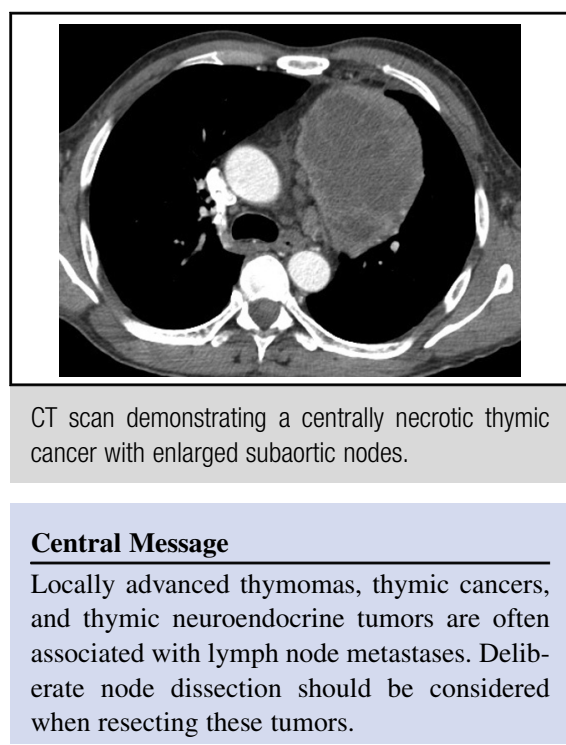

See Article page 824

whereas thymomas are more challenging to assign an accurate WHO histology. Computed tomography (CT) characteristics that suggest a locally advanced thymic tumor include large size, areas of necrosis, edge characteristics that suggest invasion, vascular invasion, elevated diaphragm, pleural nodule, heterogeneous internal density, and lymphadenopathy. Locally advanced thymomas, thymic cancers, and thymic neuroendocrine tumors require aggressive resection to achieve an R0 resection. Given the results of this study and others, ${ }^{2,3}$ it seems prudent to perform a lymph node dissection when resecting these more advanced thymic tumors to better stage the patient and to achieve an R0 resection.

Weksler and colleagues ${ }^{2}$ reported on lymph node metastases using the Surveillance, Epidemiology, and End Results database in 176 patients with thymic carcinoma $(62 \%)$ and thymic neuroendocrine tumors $(34 \%)$. In a multivariable analysis, nodal metastases were more likely in patients with thymic neuroendocrine tumors and with more advanced tumors. Nodal metastases had a significant independent adverse impact on survival (hazard ratio, 2.9; $P=.001$ ). Median survival was 47 months in patients with nodal metastases and 124 months in patients without nodal metastases $(P<.001)$. Hwang and colleagues ${ }^{3}$ reported on 131 patients who underwent lymph node dissection who had a thymoma or thymic carcinoma. Lymph node metastases were more common in patients with thymic carcinoma $(25 \%)$ than in patients with thymoma $(5 \%)$. Nodal 
metastases were associated with advanced WHO histology and advanced $\mathrm{T}$ category. Patients with nodal metastases had a significantly worse freedom from recurrence rate compared with those with pN0 $(39 \%$ vs $89 \%, P<.001)$.

Locally advanced thymic tumors should ideally undergo integrated fluorodeoxyglucose positron emission tomography (FDG PET) imaging. A recent study reported on accuracy of staging thymic tumors with whole-body magnetic resonance imaging (MRI), FDG PET/MRI, integrated FDG PET/CT, and conventional imaging examinations. Diagnostic accuracy of the $\mathrm{N}$ category was higher with PET/MRI (94\%) and MRI (94\%) compared with conventional CT imaging $(81 \%){ }^{4}$ Clearly, thymic tumors with nodal metastases have a worse prognosis, which suggests the need for systemic therapy. Induction therapy may be given first if nodal metastases are identified or the tumor is clearly T3 or T4. Systemic adjuvant therapy also may be given for patients who are found to be node positive after resection. Which strategy is better is unknown. Girard and Merveilleux du Vignaux ${ }^{5}$ recently reviewed the role of systemic treatment for thymic malignancies, highlighting newer promising drugs. Adjuvant radiation is typically administered to patients after resection of a thymic carcinoma. Lim and colleagues ${ }^{6}$ recently reported a propensity-matched SEER database study of 312 patients with thymic carcinoma, of whom 184 had postoperative radiation. ${ }^{6}$ The receipt of postoperative radiation was associated with an improved 5-year survival $(P=.007)$.

\section{References}

1. Fang W, Wang Y, Pang L, et al. Lymph node metastasis in thymic malignancies: a Chinese multicenter prospective observational study. J Thorac Cardiovasc Surg. 2018;156:824-33.e1.

2. Weksler B, Holden A, Sullivan JL. Impact of positive nodal metastases in patients with thymic carcinoma in thymic neuroendocrine tumors. J Thorac Oncol. 2015; 10:1642-7.

3. Hwang Y, Park IK, Park S, Kim ER, Kang CH, Kim YT, et al. Lymph node dissection in thymic malignancies: implication of the ITMIG node map, TNM stage classification and recommendations. J Thorac Oncol. 2015;11:108-14.

4. Ohno Y, Kishida Y, Seki S, Koyama H, Yui M, Aoyagi K, et al. Comparison of interobserver agreement and diagnostic accuracy for IASLC/ITMIG thymic epithelial tumor staging among co- registered FDG PET/MRI, whole-body MRI, integrated FDG-PET/CT, and conventional imaging examination with and without contrast media administrations. Acad Radiol. January 22, 2018 [Epub ahead of print].

5. Girard N, Merveilleux du Vignaux C. Systemic treatment for thymic malignancies. Curr Opin Oncol. 2017;29:112-7.

6. Lim YJ, Song C, Kim JS. Improved survival with postoperative radiotherapy in thymic carcinoma: a propensity matched analysis of Surveil lance, Epidemiology and End Results (SEER) database. Lung Cancer. 2017;108:161-7. 